\title{
Hematoma subdural crónico asociado a quiste aracnoideo. Presentación de 12 casos
}

\author{
M. Gelabert-González; D. Castro-Bouzas; A. Arcos-Algaba; J.M. Santín-Amo; L. Díaz-Cabanas; R. Serramito- \\ García; E. Arán-Echabe; A. Prieto-González y A. García-Allut \\ Servicio de Neurocirugía. Hospital Clínico Universitario de Santiago de Compostela. Departamento de Cirugía. Universidad de Santiago de \\ Compostela.
}

\section{Resumen}

Objetivos. Analizar las características clínicas, neurológicas y resultado neurorradiológico de una serie de pacientes con quiste aracnoideo asociado a un hematoma subdural crónico.

Pacientes y método. Revisamos retrospectivamente 12 casos de pacientes con quiste aracnoideo que presentaron como complicación un hematoma subdural crónico.

Resultados. Entre enero de 1984 y abril de 2008, 12 pacientes $(0.9 \%)$ de 1.253 casos con hematoma subdural crónico presentaban un quiste aracnoideo intracraneal. La localización de los quistes fue en 10 casos en la fosa temporal y los otros dos en la convexidad. Los síntomas de presentación fueron cefalea en 6 pacientes $(50 \%)$, seguidos por convulsiones ( 3 casos). En once pacientes se realizó agujero de trépano y drenaje del HSC, la paciente de más edad fue tratada de forma conservadora. Únicamente se trataron 3 quistes aracnoideos mediante craneotomía y fenestración, con resolución completa del quiste.

Conclusiones. Los pacientes con quiste aracnoideo, sobre todo si esta localizado en la fosa temporal, tienen un mayor riesgo de sufrir un hematoma subdural crónico. La primera opción terapéutica es el drenaje del hematoma subdural y si persiste la sintomatología deberá tratarse el quiste aracnoideo.

PALABRAS CLAVE: Espacio subdural. Quiste aracnoideo. Hematoma subdural crónico. Resonancia magnética. Traumatismo craneal.

Chronic subdural hematoma associated with arachnoid cyst. Report of 12 cases

Summary

Introduction. We designed this study to investigate

Recibido: 3-10-09. Aceptado: 5-11-09 the clinical, neurological, and radiological outcome of patients with chronic subdural hematoma related to an intracranial arachnoid cysts.

Patients and methods. Medical records of 12 cases of patients with arachnoids cyst complicated with chronic subdural hematoma were retrospectively reviewed.

Results. Between January 1984 and April 2008, 12 patients $(0.9 \%)$ of 1.253 cases of chronic subdural hematoma surgically treated in our hospital had associated arachnoid cyst. Arachnoid cysts were located in the middle fossa (10 cases) and convexity ( 2 cases). The most frequent symptom was headache (6 cases), followed by seizures (3 cases). Eleven patients underwent burr hole and drainage; the oldest patient was treated conservatively.

Conclusions. Patients with AC, especially when these are located in temporal fossa, appear to harbour a life-long risk of contracting subdural hematoma. Hematoma evacuation is adequate at first operation and if arachnoid cyst is symptomatic or preoperative symptoms persist, additional arachnoid cyst surgery should be considered.

KEY WORDS: Arachnoid cyst; Chronic subdural hematoma; Head injury; Magnetic resonance imaging; Subdural space.

Introducción

Los quistes aracnoideos (QA) son lesiones benignas intracraneales que están formadas por una membrana aracnoidea que contiene en su interior un fluido similar en sus características químicas al líquido cefalorraquídeo (LCR). Representan hasta el $1 \%$ de todas las lesiones intracraneales ocupantes de espacio y la mitad aproximadamente se localizan en la fosa temporal ${ }^{7,8,13}$. El hematoma subdural crónico es una lesión hemorrágica habitualmente de naturaleza traumática, característico de personas de edad

Abreviaturas. AC: Arachnoid cyst. HSC: hematoma subdural crónico. LCR: líquido cefalorraquídeo. QA: quistes aracnoideos. 
avanzada o con trastornos de la coagulación ${ }^{6}$.

La asociación entre HSC y QA es conocida de antiguo, sin embargo, en la literatura existen numerosos casos clínicos aislados que recogen esta relación y pocas series amplias que los analicen en detalle ${ }^{12,13,22}$.

Realizamos un estudio sobre los 12 casos de HSC asociados a quiste aracnoideo intracraneal, diagnosticados y tratados en nuestro servicio en un período de 25 años.

\section{Pacientes y método}

Entre Enero de 1984 y Abril de 2008, hemos intervenido en nuestro servicio de Neurocirugía 1.253 pacientes afectados de hematoma subdural crónico y de los cuales 12 casos presentaban asociado un quiste aracnoideo intracraneal. Analizamos además de las características demográficas, las formas de presentación clínica, la situación clínica al ingreso aplicando a los pacientes según la escala de Markwalder $^{14}$ (grado 0: sin déficit neurológico; grado 1: síntomas mínimos como cefalea; grado 2: desorientado, déficit neurológico ligero; grado 3: paciente en coma pero con respuesta ante estímulos, déficit neurológico grave; grado 4: coma profundo, sin respuesta motora, postura de descerebración o decorticación), el tipo y la localización del quiste aracnoideo, el tratamiento practicado y el seguimiento de los mismos que fue en todo ellos superior a 1 año.

\section{Resultados}

Los 12 pacientes estudiados constituyen el $0.9 \%$ de los HSC operados en un período aproximado de 25 años.

Edad y sexo. La serie consta de 9 varones y 3 mujeres con una edad media de 48.9 años (rango 16-88), siendo de 41.7 años para los varones y de 60 para las mujeres.

Forma de presentación. La cefalea fue el primer síntoma en 6 pacientes $(50 \%)$, seguida de 3 casos $(25 \%)$ que debutaron con crisis comicial. Los otros 3 pacientes se manifestaron con déficit focal (hemiparesia: 2 casos) y deterioro cognitivo en el restante. El intervalo transcurrido entre el primer síntoma y el diagnóstico osciló entre 0 y 34 días (medía: 11.8 días); siendo inferior a 24 horas en los pacientes que debutaron con una crisis epiléptica y de 10.1 días para los restantes.

Exploración y grado clínico. Seis pacientes (50\%) se encontraban en el momento del diagnóstico, en grado 2 de

Tabla 1

Datos generales de la serie

\begin{tabular}{|c|c|l|l|c|l|l|l|}
\hline Caso & $\begin{array}{l}\text { Edad- } \\
\text { Sexo } \\
\text { síntoma }\end{array}$ & $\begin{array}{c}\text { Intervalo } \\
\text { síntoma- } \\
\text { diagnóstico }\end{array}$ & $\begin{array}{c}\text { Grado de } \\
\text { Markwalder }\end{array}$ & $\begin{array}{l}\text { Localización } \\
\text { del quiste } \\
\text { aracnoideo }\end{array}$ & Tratamiento & $\begin{array}{l}\text { Evolución } \\
\text { del quiste }\end{array}$ \\
\hline 1 & $35-\mathrm{V}$ & Cefalea & $15 \mathrm{~d}$ & 2 & Silviano I & $\begin{array}{l}\text { Trepano+ } \\
\text { Fenestración }\end{array}$ & $\begin{array}{l}\text { Resolución } \\
\text { completa }\end{array}$ \\
\hline 2 & $64-\mathrm{V}$ & $\begin{array}{l}\text { Deterioro } \\
\text { cognitivo }\end{array}$ & $14 \mathrm{~d}$ & 2 & Frontal I & $\begin{array}{l}\text { Trepano }+ \\
\text { Fenestración }\end{array}$ & $\begin{array}{l}\text { Resoución } \\
\text { completa }\end{array}$ \\
\hline 3 & $19-\mathrm{V}$ & Cefalea & $2 \mathrm{~d}$ & 4 & Silviano D & Trepano & Sin cambios \\
\hline 4 & $41-\mathrm{M}$ & Hemiparesia & $3 \mathrm{~d}$ & 2 & Silviano I & Trepano & Sin cambios \\
\hline 5 & $51-\mathrm{V}$ & Crisis & 0 & 2 & Silviano I & Trepano & Sin cambios \\
\hline 6 & $49-\mathrm{V}$ & Cefalea & $10 \mathrm{~d}$ & 1 & Silviano D & Trepano & Sin cambios \\
\hline 7 & $16-\mathrm{V}$ & Crisis & 0 & 1 & Silviano I & Trepano + & $\begin{array}{l}\text { Resolución } \\
\text { completa }\end{array}$ \\
\hline 8 & $52-\mathrm{M}$ & Cefalea & $34 \mathrm{~d}$ & 1 & Silviano I & Trepano & Sin cambios \\
\hline 9 & $43-\mathrm{V}$ & Crisis & 0 & 2 & Silviano I & Trepano & Sin cambios \\
\hline 10 & $38-\mathrm{V}$ & Hemiparesia & $2 \mathrm{~d}$ & 2 & Silviano D & Trepano & Sin cambios \\
\hline 11 & $88-\mathrm{M}$ & Cefalea & $7 \mathrm{~d}$ & 1 & Frontal I & Conservador & Resolución \\
\hline 12 & $61-\mathrm{V}$ & Cefalea & $7 \mathrm{~d}$ & 1 & Silviano I & Trepano & Sin cambios \\
\hline
\end{tabular}

d: días; D: derecho; I: izquierdo; M: mujer; V: varón 


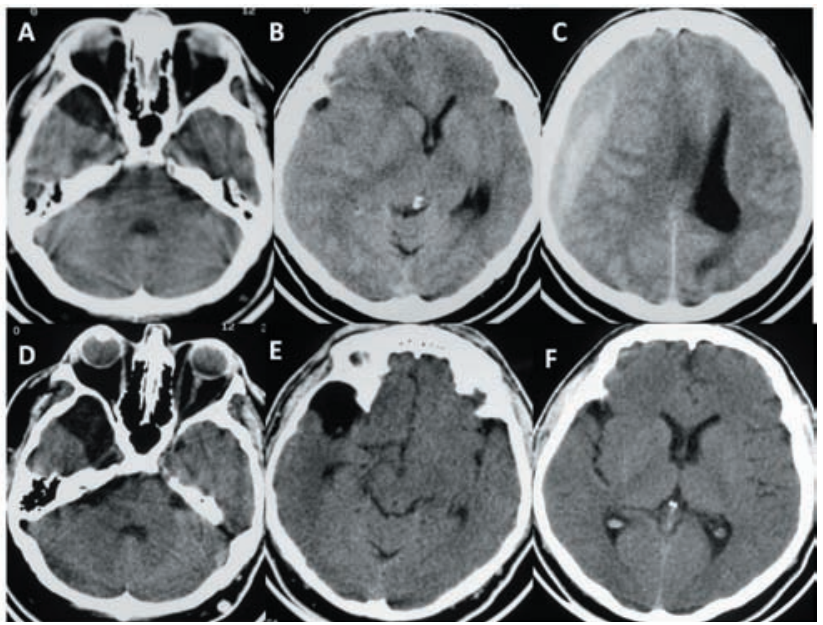

Figura 1. (A-C): TC al ingreso mostrando el QA y hematoma subdural crónico. (D-F): TAC a los 6 meses de la cirugía mostrando la persistencia del $Q A$.

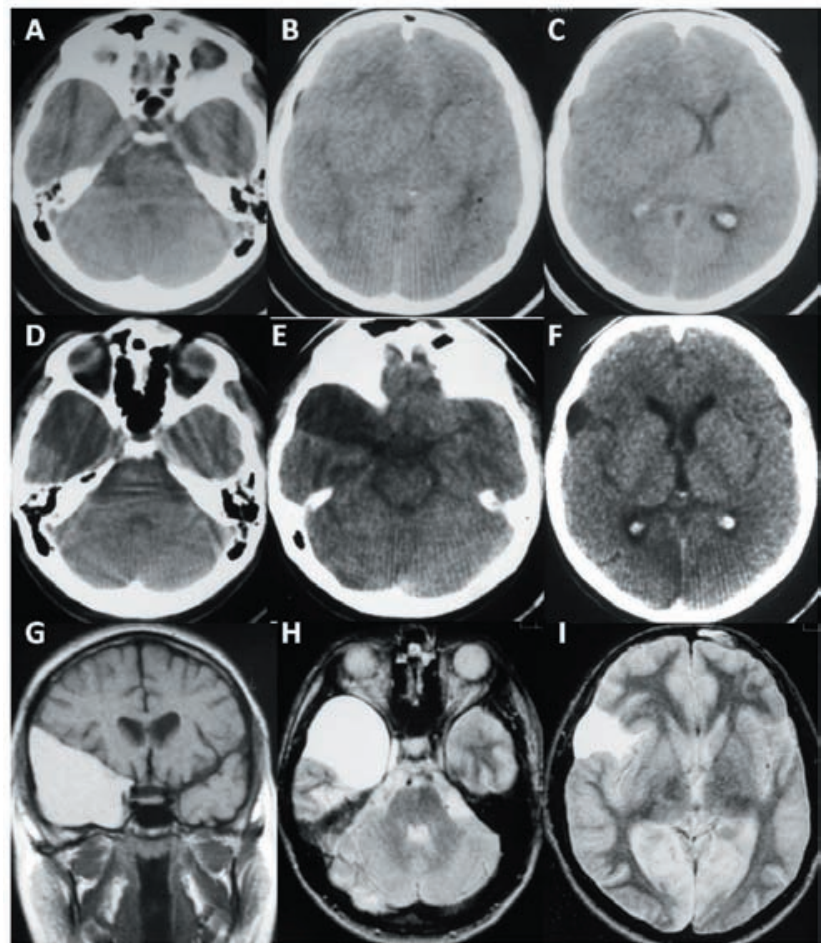

Figura 2. (A-C): TC al ingreso mostrando un HSC isodenso, el QA esta colapsado. (D-F): TC a los 15 días de la cirugía mostrando el QA. (G-I): RM al año de la cirugía.

Markwalder, es decir presentando afectación de funciones cerebrales superiores y déficit neurológico, 5 casos $(41.6 \%)$ presentaban cefalea sin afectación focal (grado 1) y únicamente un paciente, se encontraba en coma profundo con signos de enclavamiento temporal en el momento del diagnostico (grado 4).

Localización del quiste aracnoideo. Excepto dos casos

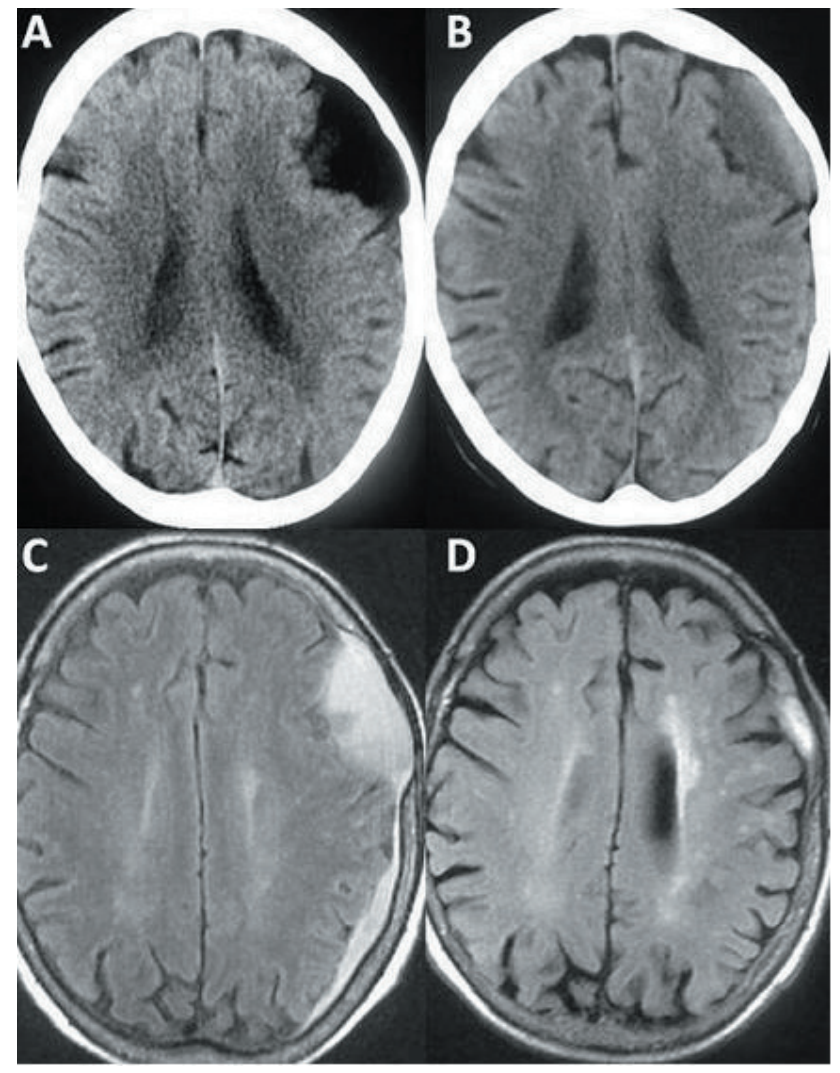

Figura 3. (A): TC realizado 4 años antes del ingreso. (B): $T C$ practicado tras el traumatismo mostrando cambio de densidad en el $Q A$. (C): $R M$ realizada 7 días tras el traumatismo, se observa el pequeño hematoma subdural. (D): RM al año mostrando la resolución del QA y del HSC.

de quiste frontal los restantes pacientes presentaban quistes silvianos, siendo 6 tipo I y 4 tipo II de Galassi ${ }^{5}$. Nueve quistes eran izquierdos y 3 derechos (Figura 1-3).

Tratamiento. En once casos se realizó un agujero de trépano, lavado del espacio subdural con suero fisiológico e implantación de un drenaje subdural mantenido entre 24-72 horas. La paciente de más edad de la serie, que se encontraba paucisintomática se decidió tratamiento conservador, evolucionando hasta la resolución del hematoma y del quiste (Figura 3).

Evolución. Todos los pacientes se recuperaron satisfactoriamente tras el tratamiento recibido, con resolución completa del hematoma subdural en todos los casos. Al alta, 11 pacientes se encontraban asintomáticos (grado 0 de Marwalder) y el paciente número 1 desarrolló un trastorno campimétrico derivado de un infarto occipital derecho como consecuencia de la hipertensión intracraneal y de la hernia tentorial.

Tratamiento de los quistes. De todos los quistes diagnosticados, únicamente fueron tratados 3 , mediante cráneotomía y fenestración el mismo. En los restantes 9 pacientes 
no se realizó tratamiento especifico (6 casos no se consideró cirugía y 3 pacientes rechazaron el tratamiento) practicándose seguimiento clínico y radiológico de los quistes. Ninguno de ellos ha tenido más problemas relacionados con la lesión.

\section{Discusión}

Los quistes aracnoideos son lesiones ocupantes de espacio de origen congénito que pueden localizarse en cualquier punto del sistema nervioso central aunque en general la fosa craneal media es el sitio más común, especialmente en la cisura silviana ${ }^{17}$. Son característicos de la edad pediátrica donde representan el 1\% de todas las lesiones expansivas intracraneales. Aunque muchos de los QA no presentan sintomatología durante la infancia y pueden diagnosticarse en la edad adulta, bien por que se hacen sintomáticos o por ser hallazgos incidentales ${ }^{8,13}$.

La sintomatología de los QA está en relación directa con la edad de los pacientes, en la población infantil suelen provocar deformidades del cráneo cuando asientan en la convexidad de los hemisferios cerebrales o incremento de la presión intracraneal relacionada con la obstrucción en la circulación del líquido cefalorraquídeo cuando se sitúan en la línea media ${ }^{10}$. En niños de más edad y pacientes adultos, los QA pueden manifestarse de formas muy diferentes, habitualmente relacionadas con el progreso en el volumen del quiste o con diversas complicaciones asociadas como es la hemorragia espontánea intraquística, la ruptura espontánea o traumática hacia el espacio subdural o su relación con el desarrollo de hematomas subdurales s,13,15,16. $^{7}$.

El hematoma subdural crónico es una colección de sangre en diversas fases de degradación localizada en el espacio subdural que crece de forma lentamente progresiva ocasionando una sintomatología, habitualmente de bajo nivel. Es característico de la población anciana donde se ve favorecido su desarrollo por la involución senil del cerebro, la ingesta de fármacos que alteran la coagulación sanguínea y una mayor tasa de accidentes, habitualmente banales, que condicionan el inicio de una serie de mecanismos que conducen a la formación del hematoma ${ }^{6}$. La aparición de un HSC en pacientes jóvenes $(<50$ años) debe hacernos pensar siempre en la posibilidad de que exista algún factor sobreañadido para el desarrollo del mismo, entre los que se encuentran las válvulas de derivación ventricular, fístulas espontáneas de LCR, etc. ${ }^{16}$ o un quiste aracnoideo. En nuestra serie general de HSC la edad media de los pacientes fue de 72.7 años ${ }^{9}$, significativamente más alta que la correspondiente a los pacientes que tenían asociado un QA que fue, como hemos visto de 48.9.

La relación entre HSC y QA se conoce desde antiguo en la literatura neuroquirúrgica aunque no hay una explica- ción satisfactoria para justificar esta asociación. Page et a ${ }^{19}$ propusieron 2 teorías: a) tras un trauma craneal se producirían cambios en el flujo del LCR que llevarían a facilitar la rotura de venas puente o de vasos en la pared del quiste, y b) al tener los QA menos distensibilidad que el parénquima cerebral, favorecería que tras un traumatismo de poca intensidad las venas puente se rompiesen con mayor facilidad, ya sea ipso o contralateralmente, produciéndose, así, un hematoma subdural.

Haines et $\mathrm{al}^{11}$ en un estudio con microscopia electrónica de las membranas meníngeas, describe unas células en el reborde dural que se extienden desde la duramadre y se adhieren firmemente a la aracnoides, delimitando un espacio virtual que denomina "compartimento subdural". Este espacio permanece sellado y puede abrirse, ante un traumatismo, formándose el hematoma subdural. Servadei et $\mathrm{a}^{23}$ comparten esta opinión y en 3 de sus casos no relacionan la ruptura de venas puente con la patogenia del HSC, pero encontraron que el origen del sangrado se encontraba entre la duramadre y la membrana del quiste.

Recientemente Wester y Helland ${ }^{24}$ encuentran en su serie de 241 casos de HSC 11 casos de quiste aracnoideo asociado al hematoma (4.6\%), todos ellos de localización temporal y apoyan estas dos teorías basándose en observaciones realizadas sobre los mismos. Su primera observación es que en los QA de la fosa media, las fuerzas mecánicas que se originan durante el traumatismo, ocasionarían un aumento en la separación entre duramadre y membrana del quiste, favoreciendo la ruptura de algún vaso de la membrana. Este mecanismo también explicaría la asociación entre HSC y QA a nivel espinal ${ }^{18}$. Su segunda observación intraoperatoria está en la línea de la teoría de Page et $\mathrm{al}^{19}$, es decir, la membrana del QA cubre el área donde las venas puentes silvianas atraviesan el espacio subdural aumentando su vulnerabilidad ante cualquier tipo de traumatismo. Esta relación entre venas puente y membranas de un QA no se produce en otras localizaciones de la convexidad, lo que explicaría la alta incidencia de hematomas en la región temporal.

Domenicucci et $\mathrm{al}^{3}$ pudieron observar que en estudios precoces de neuroimagen practicados a pacientes con hematomas subdurales que los hematomas están situados en el "compartimento intradural".

En ambas teorías se asume que las dos lesiones tienen una relación de vecindad, como ocurre en la mayoría de los casos publicados, sin embargo, existen también en la literatura casos descritos donde el HSC y el QA asientan en distinto hemisferio cerebral ${ }^{21}$.

El tratamiento de estos pacientes es controvertido, algunos autores defienden el tratamiento exclusivo del hematoma subdural (agujero de trépano y drenaje) basándose en el hecho de que tras la evacuación, muchos quistes tienden a remitir de forma espontánea, sobre todo si el QA 
era asintomático ${ }^{4,20,25}$. Por el contrario, existe una opinión mayoritaria del tratamiento simultáneo o sucesivo de ambas lesiones mediante una craneotomía que permita la evacuación del hematoma y la fenestración de las membranas del quiste ${ }^{18,23}$ o evacuación del hematoma e inserción de una derivación cisto-peritoneal ${ }^{1,22}$. En nuestra serie de los 11 pacientes sometidos a cirugía del HSC únicamente se intervinieron 2 pacientes mediante craneotomía, en otros 3 casos se propuso microcirugía debido al volumen del quiste pero ésta fue rechazada. Aunque algunos estudios proponen el tratamiento esteroideo en pacientes con $\mathrm{HSC}^{2}$ sólo lo empleamos en la paciente más anciana de la serie, consiguiéndose un buen resultado tanto con resolución del hematoma y del quiste, probablemente debido a que el quiste aracnoideo se rompió en el espacio subdural por efecto del traumatismo.

\section{Bibliografía}

1. Arai, H., Sato, K., Wachi, A., Okuda, O., Takeda, N.: Arachnoid cysts of the middle cranial fossa: experience with 77 patients who were treated with cystoperitoneal shunting. Neurosurgery 1996; 39: 1108-1112.

2. Delgado-López, P.D., Martin-Velasco, V., Castilla-Díez, J.M., Rodríguez-Salazar, A., Galacho-Harriero, A.M., Fernández-Arconada, O.: Dexamethasone treatment in chronic subdural haematoma. Neurocirugía 2009; 20: 346-359.

3. Domencucci, M., Strzelecki, J.W., Delfini, R.: Acute posttraumatic subdural hematoma: "intradural" computed tomographic appearance as a favourable prognostic factor. Neurosurgery 1998; 42: 51-55.

4. Domenicucci, M., Russo, N., Giugni, E., Pierallini, A.: Relationship between supratentorial arachnoid cyst and chronic subdural hematoma: neuroradiological evidence and surgical treatment. J Neurosurg 2009; 110: 1250-1255.

5. Galassi, E., Tognetti, F., Geist, G, et al.: CT scan and metrizamide CT cisternography in arachnoid cysts of the middle cranial fossa: classification and pathophysiological aspects. Surg Neurol 1982; 17: 363-369 .

6. Gelabert, M., Fernández, J.M., López, E., García, A.: Hematoma subdural crónico en el paciente mayor de 80 años. Neurocirugía 2001; 12: 325-330.

7. Gelabert, M., Fernández, J.M., Cutrín, J.M., García, A., Martínez, R.: Arachnoid cyst rupture with subdural hygroma. Report of three cases and literature review. Child's Nervous System 2002; 18: 609-613.

8. Gelabert, M.A., Prieto, J., González, A.G., Pravos, I., Abdulkader, A., García. A.: Quiste aracnoideo intraventricular en el adulto. Rev Neurol 1997; 25: 10951098.

9. Gelabert-González, M., Iglesias-Pais, M., García-Allut, A., Martínez-Rumbo R.: Chronic subdural haematoma: surgi- cal treatment and outcome in 1000 cases. Clin Neurol Neurosurg 2005; 107: 223-229.

10. Gómez-Escalonilla, C.I., García-Morales, I., GalánDávila, L., et al.: Quistes aracnoideos intracraneales. Estudio de una serie de 35 casos. Rev Neurol 2001; 33: 305-310.

11. Haines, D.E., Harkey, H.L., Al-Mefty, O.: The "subdural" space: A new look at an outdated concept. Neurosurgery 1993; 32: 111-120.

12. Iglesias, A., Arias, M., Meijide, F., Brasa, J.: Quiste aracnoideo complicado con hemorragia intraquística y hematoma subdural espontáneo: hallazgos en resonancia magnética. Radiología 2006; 48: 245-248.

13. Martín, S.: Quistes aracnoideos revelados por un síndrome de hipertensión endocraneal tras traumatismo craneal. Neurocirugía 1990; 1: 223-225.

14. Markwalder, T.M., Steinsiepe, K.F., Rohner, M., Reichenbach, W., Markwalder, H.: The course of chronic subdural haematomas after burr-hole craniostomy and closed-system drainage. J Neurosurg 1981; 55: 390-396.

15. Mayordomo-Colunga, J., Anes, G., Los Arcos, M., Álvarez, F.: Hematoma subdural subagudo asociado a quiste aracnoideo. An Pediatr (Barc) 2009; 70: 83-84.

16. Mori, K., Yamamoto, T., Horinaka, N, Maeda, M.: Arachnoid cyst is a risk factor for chronic subdural hematoma in juveniles: twelve cases of chronic subdural hematoma associated with arachnoid cyst. J Neurotrauma 2002; 19: 1017-1027.

17. Muñoz, A., Benito-León, J., Carrasco, A., Ayerbe, J., Domínguez, J., Cordobés, F.: Hematoma subdural crónico asociado a quiste aracnoideo. Neurología 1996; 11: 263267.

18. Muthukumar, N.: Anterior cervical arachnoid cyst presenting with traumatic quadriplegia. Childs Nerv Syst 2004; 20: 757-760.

19. Page, A., Paxton, R.M., Mohan, D.: A reappraisal of the relationship between arachnoid cyst of the middle cranial fossa and chronic subdural hematoma. J Neurol Neurosurg Psychiatry 1987; 50: 1001-1007.

20. Parsch, C., Krau, J., Hoffman, E., Meixensberger, J., Roosen, K.: Arachnoid cysts associated with subdural hematomas and hygromas. Follow-up, and review of the literature. Neurosurgery 1997; 40: 483-490.

21. Pillai, P., Menon, S.K., Manjooran, R.P., Kariyattil, R., Pillai, A.B., Panikar, D.: Temporal fossa arachnoid cyst presenting with bilateral subdural hematoma following trauma: two cases reports. J Med Case Reports 2009; 3: 5357.

22. Rogers, M.A., Klug, G.L., Siu, K.H.: Middle fossa arachnoid cysts in association with subdural haematomas. A review and recommendations for management. Br J Neurosurg 1990; 4: 497-502.

23. Servadei, F., Vergani, G., Fratarelli, M., Pasini, A., 
Arista, A., Fagioli, L.: Arachnoid cyst of middle cranial fossa and ipsilateral subdural hematoma: diagnostic and therapeutics implications in three cases. Br J Neurosurg 1993; 7: 249253.

24. Wester, K., Helland, C.A.: How often do chronic extracerebral haematomas occur in patients with intracranial arachnoid cysts? J Neurol Neurosurg Psychiatry 2008; 79: 72-75.

25. Zhang, H., Zhang, J., Chen, G.: Chronic subdural hematoma associated with arachnoid cyst: report of two cases. Chin Med J 2007; 120: 2339-2340.
Gelabert-González, M.; Castro-Bouzas, D.; Arcos-Algaba, A.; Santín-Amo, J.M.; Díaz-Cabanas, L.; Serramito-García, R.; Aran-Echabe, E.; Prieto-González, A.; García-Allut, A.: Hematoma subdural crónico asociado a quiste aracnoideo. Presentación de 12 casos. Neurocirugía 2010; 21: 222-227

Correspondencia: Miguel Gelabert-González. Servicio de Neurocirugía. Hospital Clínico Universitario. A Choupana. 15706 Santiago de Compostela.

miguel.gelabert@usc.es 